Cahiers de recherches médiévales

Journal of medieval studies

3 | 1997

Espace vécu, mesuré, imaginé

\title{
Dangereux Occident
}

Le voyage de Léon de Rozmital jusqu'à Saint-Jacques de Compostelle (1465-1466)

\section{Françoise Michaud-Fréjaville}

\section{(2) OpenEdition \\ 12 Journals}

Édition électronique

URL : https://journals.openedition.org/crm/2458

DOI : $10.4000 / \mathrm{crm} .2458$

ISSN : 1955-2424

Éditeur

Honoré Champion

Édition imprimée

Date de publication : 15 janvier 1997

Pagination : 57-69

ISSN : 1272-9752

\section{Référence électronique}

Françoise Michaud-Fréjaville, «Dangereux Occident », Cahiers de recherches médiévales [En ligne], 3 |

1997, mis en ligne le 04 février 2008, consulté le 15 décembre 2022. URL : http://

journals.openedition.org/crm/2458; DOI : https://doi.org/10.4000/crm.2458 


\section{RM}

\section{Dangereux occident, le voyage de Léon de Rozmital jusqu'à Saint- Jacques de Compostelle. (1465-1466)}

Leon, lord of Rozmital, brother of the queen of Bohemia, leaves Prague with about twenty riders to travel, to see the world and eventually to enter into negotiations with a view to help the king of Bohemia battling with the pope. This travel is a long initiatic narration in a world full of dangers, outside and also inside, which are useful for measuring merits of the men and of the group. This dangerous world, by its own species and also through the ignorance of those who face it, discloses to each other what he is and so becomes a deep interior travel which refers to the space so seized.

Le 26 novembre 1465, une petite vingtaine de cavaliers, cinquante deux chevaux et un chariot quittèrent Prague pour Saint-Jacques de Compostelle. Il s'agissait du propre frère de la reine de Bohème. Léon (Lev), seigneur de Rozmital et de Blatna, de trois chevaliers, et d'une suite de compagnons et de serviteurs. Le propos était à la fois politique, religieux et romanesque : parcourir le monde en tournoyant et joutant, visiter les lieux de culte et recevoir les bienfaits des reliques insignes conservées en Occident, sonder les cours royales et princières sur le soutien que celles-ci pourraient apporter au roi de Bohême, Georges de Podiebrad (14571471) dans le conflit qui l'opposait au pape Paul II sur la question hussite. Les raisons secrètes expliquent le trajet incongru des «pèlerins »: au lieu de se diriger droit vers Compostelle, par Nuremberg, Ulm. Constance, Genève, Lyon et la Via Podensis (du Puy), ils rendirent visite en traversant l'Empire, au duc de Bourgogne, puis franchirent la Manche pour se présenter à Edouard IV d'Angleterre. Revenus sur le continent, ils firent un crochet après avoir été reçus par le roi René en Anjou, et cherchèrent Louis XI sur les bords de la Loire. Passés en Espagne, ils tentèrent de se concilier les deux partis castillans. et durent descendre jusqu'à Ségovie pour être admis en présence d'Henri IV de Castille, puis ils pénétrèrent au Portugal, y virent le souverain Alphonse $\mathrm{V}$, et, enfin, arrivèrent à Saint-Jacques. Le voyage fut fort long, ils touchèrent le but théorique de leur déplacement le 12 août 1466 , près de neuf mois après avoir franchi les portes de Prague. Nous ne nous occupons pas ici du retour.

Ce long cheminement dans une chrétienté troublée (pas un seul des pays traversés qui ne soit alors secoué par des crises politiques graves), deux récits l'ont rapporté, celui en langue tchèque de Venceslas Schaschek (de Birkova?), un jeune homme, sans doute d'origine noble, dont on sait peu de choses, sinon ce qu'il laisse transparaître dans son texte, et un second, en allemand, de Gabriel Tetzel, un éminent bourgeois de Nuremberg, expert en joute, que le prince Léon avait convié à l'accompagner ${ }^{1}$. Le texte tchèque a disparu, hélas, dans sa forme vernaculaire, mais

\footnotetext{
' Bayerische Staatsbibliothek, Cod. Germ. 1279, fol. 128-178v. Das bömischen Hern Leo's von Rozmital Ritter -, Hof -, und Pilgerreise durch die Abendlande, 1465-1467, beischrieben
}

Cahiers de Recherches Médiévales (XIII'-XV s.), 3. 1997 
a été traduit en 1577, dans un latin ultra-classique par un humaniste, Stanislas Pawlowsky, qui devint évêque d'Olomuc en $1579^{2}$. Les discussions sur ce qui a pu être interprété par le traducteur ont été nombreuses, mais, finalement, elles n'ont pas vraiment d'influence sur l'analyse que je tente aujourd'hui du rapport rédigé par l'écuyer-page et peut-être boute-en-train sur l'équipée de nos seigneurs aventureux.

Les deux sources montrent, en effet, des tempéraments différents dont je ne pousse pas trop loin pourtant l'opposition et les caractérisations, de crainte de faire dire aux textes plus qu'ils n'en peuvent trahir. Cependant, il y a entre les deux compagnons une différence d'âge, l'un n'a jamais rien vu et l'autre a déjà voyagé, a fréquenté quelques grandes villes et cours princières. L'un se fait naif, " j'aurai voulu voir », «j'ai vu de mes yeux », « si j'ai jamais vu l'équivalent... », il s'émerveille des marées et de l'habileté des matelots, hume l'air, contemple les fleurs; l'autre blasé, manie davantage l'ellipse : des étuves de Bruges « il y aurait beaucoup à dire... ». Le premier admire les trésors du duc de Bourgogne qui lui sont montrés et le second estime froidement le prix de ces objets, comme un commissaire priseur, le marchand l'emportant ici sur le tournoyeur! Tous deux cependant sont des familiers du prince, le suivent - pas toujours de très près ${ }^{3}$ - dans ses visites auprès des rois et des évêques et, à quelques exceptions près, ont vu les mêmes scènes, dont ils nous livrent des rapports subtilement éclairés par leurs personnalité propres.

Pour honorer notre amie Christiane Deluz, en la suivant sur les pas des voyageurs, sans trop dévier de mes travaux passés ou paraître révéler des nouveautés sur une littérature sur laquelle j'aurais plutôt tout à apprendre ${ }^{4}, j^{\prime}$ 'ai choisi de privilégier le texte de Schaschek dont il m'a paru qu'il était, certes, un rapport de voyage et de pèlerinage, mais aussi, pour lui-même, un long récit initiatique. Le monde dans lequel le jeune homme se déplace se révèle non seulement sous sa forme visible voire anecdotique, mais aussi sous ses aspects profonds, où les dangers servent à mesurer la valeur des individus et du groupe.

Triompher des obstacles, au prix de la révélation de ses propres capacités, mais aussi, céder, reconnaître ses limites pour mieux se vaincre, telles sont les leçons qu'avaient reçues nos bouillants et fiers jeunes gens quand, pieds nus et genoux en terre, ils attendirent longuement à la porte du tombeau de Saint-Jacques

von zweien seiner Beigleiter, dans Bibliothek des litterarischen Vereins in Stuttgart, VII, 1844.

${ }^{2}$ Commentarius brevis et iucundus itineris atque perigrinationis pietatis et religionis causa susceptae ab Illustri et Magnifico Domino, Domino Leone, libero barone de Rosmital et Blatna, éd. K. Hrdina, Prague, 1951. Une traduction anglaise, complète du récit de Tetzel et lacunaire de celui de Schaschek, a été donnée par M. Letts, The travels of Leo of Rozmital trough Germany, Flandres, England, France, Spain, Portugal and Italy, 1465-1467, Cambridge, 1957 (The Hakluyt society, second series. CVIII). Mes remerciements vont à Frantisek Smahel à Prague qui m'a très aimablement procuré le texte édité par Hrdina, à A. Vantuch, et, surtout, à Madame E. Krutova. à Brno, qui m'a fait comprendre l'intérêt de ce récit de voyage.

${ }_{3}$ «En ce lieu (dans la propre chambre de Philippe le Bon) autant que j'ai pu le voir, après s'être entretenu avec lui, le prince prit congé de monseigneur ", Commentarius, p. 22.

$4 \mathrm{~J}$. Richard, Les récits de voyages et de pélerinages. Turnhout, 1981 (Typologie des sources du Moyen Age, 38). 
de se voir relevés de l'excommunication que leurs imprudentes, mais inévitables, relations locales leur avait value ${ }^{5}$ ! Bardés de sauf-conduits, armés jusques aux dents, beaux comme des dieux, forts comme des boeufs, les voici humblement et patiemment soumis aux coups de ceinture du légat pontifical. Rude fin d'apprentissage au bout d'une route pleine d'imprévu.

Sans doute le chemin fut-il parfois plein de délices. Que de jolies dames et de bons repas : «nous menions, en vérité, vie joyeuse et mondaine » dit Tetzel du séjour à Bruges. Ils ont apprécié l'étonnante et charmante habitude des hôtesses anglaises d'accueillir les voyageurs par un baiser ${ }^{6}$. Ils ont vu les nonnes aristocratiques et douées pour la danse du monastère de Las Huelgas ${ }^{i}$. Les gens de Bohême découvrirent successivement la chasse au furet ${ }^{8}$. les patins à glace ${ }^{9}$, les courses de taureaux $^{10}$, les salines ${ }^{11}$ et les orangers ${ }^{12}$. Néanmoins, quel mérite aurait-on, sinon celui de satisfaire sa curiosité et sa soif du paraître si tout se déroulait sur un air de danse ? Non, les dangers existent et c'est par eux que doivent passer les « ignorants » pour acquérir une véritable expérience.

\section{Les dangers de la Nature}

Partis en plein hiver, les voyageurs ne se plaignent pas du froid, ce qui n'est pas sans nous surprendre, car, au hasard des pages, nous découvrons les étangs glacés de Bruxelles, supportant les patineurs ${ }^{13}$ : mais la neige, le vent, la pluie semblent choses normales, ne perturbent pas les cheminements terrestres et ne

\footnotetext{
${ }^{5}$ Ils arrivèrent à Compostelle alors que la province était frappée d'interdit. L'archevêque, Alphonse Fonseca (1460-1512) était prisonnier de Bernard Yanez de Moscoso dont les troupes assiégeaient, en outre, la cathédrale. Dans celle-ci, la mère et le frère de l'archevêque bloquaient les chanoines réfugiés dans le triforium! Pour pouvoir entrer dans le sanctuaire, il fallait obligatoirement discuter avec Bernard. puis faire amende honorable pour ce crime devant la mère, le légat et les partisans de l'archevéque.

${ }^{6}$ "Chez eux, en effet, c'est la même chose de donner un baiser que de se serrer la main droite, on n'a pas l'habitude de se donner la main ". Commentarius, p. 37.

7 «Elles accueillirent Monseigneur et sa compagnie avec des jeux charmants et variés, comme des danses et autres choses semblables, et le conduisirent dans les jardins les plus délicieux ». idem p. 59.

${ }^{8}$ Idem, p. 20, près d'Aix-la-Chapelle.

${ }^{9}$ Idem, p. 26: Bruxelles, Schaschek regretta fort de ne pouvoir aller voir de plus près les joueurs du bassin glacé du château et ce "par quoi leurs pieds étaient soutenus et qui leur permettait de se déplacer si rapidement sur la glace".

Idem. p. 58 : Burgos.

"Idem, p. 15 : à Haile, en Wurtemberg, où l'on fait bouillir l'eau salée dans des marmites, et, p. 56. entre Valmaseda et Medina de Pumare, en Biscaye. où le soleil permet l'évaporation des eaux de rivière salée.

12 Idem, p. 69 : arbores quae paradisiaca, aurantia, limonia granataque poma ferunt, aliasque diversi generis, tum etiam herbas a me antea non visas mullibi plures quam apud eam urbem conspeximus.

${ }^{13}$ Commentarius, p. $26:$ : ils étaient vingt-huit qui se mesuraient avec une telle dextérité que je peux dire que je n'avais vu, ni entendu parler de gens aussi agiles. Telle était leur rapidité à courir et à tourner, qu'aucun élan de cheval ne pouvait les égaler ".
} 
méritent pas une mention. Il en fut tout autrement de la chaleur et du climat ibériques $^{14}$ : sur les plateaux de Castille, les chevaux épuisés ne supportaient plus leur cavalier, ceux-ci, devenus piétons, ne trouvaient nulle part à boire, « ni eau, ni vin, même à prix d'or ${ }^{15}$. Le cheval favori de Léon mourut, avant d'arriver à Burgos $^{16}$. Les montagnes arides où les roches cristallines réverbéraient le soleil étaient éprouvantes : «cette montagne, par de petites pierres transparentes illuminées par la splendeur du soleil, répercute et renvoie cette lumière comme dans un miroir, $c^{\prime}$ est très désagréable ${ }^{17}$ ". Aucun mauvais climat cependant, ne trouble autant les voyages que les traversées maritimes. Une fois passés les étonnements du terrien d'Europe centrale qui découvre les marées alternant toutes les six heures ${ }^{18}$, et les côtes plates où se confondent les horizons et les toits ${ }^{19}$, surgit la peur des élëments aqueux, lesquels, de plus, se déchaînent, à les en croire, tout exprès pour ces terriens! «On disait que personne depuis cent ans n'avait connu ce qui nous est arrivé ". Le programme en Angleterre comprenait un pèlerinage au tombeau de saint Thomas de Cantorbéry et une visite à la cour d'Edouard IV. On devait donc passer la Manche, ensuite, les rites accomplis, regagner le continent.

D'abord, il fallut pour partir réduire le train. Devant la taille et surtout le prix des bateaux, Léon renvoya les non-nobles (garda Gabriel Tetzel, bien sûr), ne conservant que les pages attitrés de chacun, et trente-six chevaux. On ne nous dit rien du chariot. Ensuite, on attendit les vents favorables à Calais, comme on les espéra au retour successivement à Poole, à Jersey, puis, enfin, à Guernesey. Apprentissage de la patience... De plus, les vents qui apparaissent favorables peuvent cacher de noirs desseins. Ce que Schaschek refuse d'abord de narrer : «nous courûmes un grand danger que je me refuse à raconter ${ }^{20}$, Tetzel, lui, le dit.

Donc, selon le bourgeois de Nuremberg:

cette nuit le vent était favorable à la traversée, aussi nous hissâmes la voile et quittâmes la terre. Mais quand on eut laissé le port et gagné la haute mer, le bateau eut une déchirure et l'eau s'engouffra, si bien que les chevaux eurent de l'eau jusqu'aux boulets. Alors, le seigneur Dieu nous envoya la bonne Fortune. Le vent tourna en bonne brise. Mais si le vent n'avait pas tourné, alors nous aurions tous été noyés.

Revenus à Calais, ils attendirent un bon vent, et un nouvel incident, encore plus terrorisant, se produisit :

14 Le thème de l'Espagne, comme région particulièrement pénible à traverser, et se différençiant en cela de tout le reste de l'Europe, a déjà été souligné à plusieurs reprises (J.A. Garcia de Cortazar, "El hombre medieval como "homo viator ". Pelegrinos y viajeros ", IV Semanas de estudios medievales, Najera, 2-6 de agosto de 1993, Logronos, 1994, p. 17).

${ }^{15}$ Commentarius, p. 59.

${ }^{16}$ Idem, p. 55 : entre Villasano et Medina de Pumare, “c'est là que mourut le cheval favori de Monseigneur, dont il espérait beaucoup qu'il vivrait très longtemps ».

${ }_{17}^{17}$ Idem, p. 56.

${ }^{18}$ Idem, p. 20 : à Malines, per eam sex horas aquae fluunt et vicissim sex horas eam siccam destituunt, vel deficiente mari.

19 Ibidem : à Lier, « les sommets des toits sont à peine visibles en raison de la hauteur des remblais ».

${ }^{20}$ Idem, p. 33. 
Monseigneur dut prendre place dans un petit canot pour être amené à la rame au navire. Alors surgit un grand vent et une tempète, si bien que nous manquâmes de sombrer et arrivâmes au bateau à grand peine. Et si le seigneur Jean (Zarhovsky) et Gabriel Tetzel (le narrateur) n'avaient pas été là pour aider, Monseigneur eût été noyé. Monseigneur et ses compagnons étaient si maltraités par les vagues, qu'ils gisaient sur le pont comme s'ils étaient morts ${ }^{21}$.

Accostage sans gloire ni panache dans cette étrange île, par une côte « toute de chaux qu'il n'est pas besoin de traiter par le feu ${ }^{22} »$.

Si l'arrivée fut peu agréable le retour fut pire, et Schaschek en retrouva sa plume. A Poole, il fallut subir huit jours d'attente pour guetter un vent soufflant vers la Bretagne ; après le départ, aux flots encore menaçants s'ajoutèrent les corsaires nous y reviendrons -, les vents contraires poussèrent les bateaux vers Jersey, où au bout de douze jours de patience les navires reprirent la mer, rencontrèrent de nouveau les garde-côtes anglais, se trouvèrent ensuite rejetés par les vents vers Guernesey : «nous sommes restés dans cette île onze jours, privés du vent qui nous porterait vers la Bretagne ${ }^{23} »$. Les voyageurs réembarquèrent par une mer et un vent favorables...

La nuit suivante, un grand coup de vent se produisit contre nous qui étions ballottés par le flot, vent qui nous emplit tous d'une grande frayeur. A vrai dire, il ne dura guère, mais s'il avait duré, il aurait submergé un grand nombre de gens. Rien n'est plus dangereux ; il arriva sur nous si inopinément que nos marins furent pris au dépourvu! Nous ne fûmes pas loin d'être tous noyés, en effet le bateau était déjà plein de l'eau qui entrait par les ouvertures. A cause de la grande force du vent les voiles ne pouvaient plus être amenées. Une fois affalées à grand peine et labeur, alors le navire, comme il le devait. commença à se redresser. En effet, en raison de la force du vent, il était penché sur l'eau. dompté. jusqu'au bord ${ }^{24}$.

Schaschek, n'admire plus, en badaud comme il le faisait à Sandwich, les «marins escaladant un mât, prédisant l'arrivée et la direction des vents et annonçant quelles voiles devaient être hissées et lesquelles amenées ${ }^{25}$ ". Il songe que la mort fut bien proche : « quand nous fûmes sortis de cette tempête, [...] tombant à genoux, nous rendîmes grâce à Dieu de nous avoir conservés saufs de tant de dangers ». En jeune étourdi, Schaschek pense surtout aux hommes. Tetzel ajoute que le mât avait cassé (est-ce ce qui a ramené la voile. ou est-ce un détail pour faire encore plus terrible?), et qu'«il y eut grand émoi également parmi les chevaux, en dessous, pendant que la tempête faisait rage. Ils se heurtèrent de part et d'autre et parfois tombèrent les uns sur les autres. Nul ne peut y croire s'il n'a pas vécu cela ». A l'arrivée, ajoute-t-il,

nous hissâmes nos chevaux hors du navire, mais ils étaient à demi morts, ils ne pouvaient plus se tenir debout, ni avancer. Monseigneur dut rester là plusieurs jours pour que les bêtes se reconstituent quelque peu.

\footnotetext{
${ }^{21}$ Des bösmischen Hern Leo's.. Reise, p. 40.

22 Commentarius, p. 34.

${ }^{23}$ Idem, p. 43.

24 Ibidem.

${ }^{25}$ Idem, p. 34.
} 
La traversée qui devait se faire en quatre jours en avait pris dix-sept. Les seigneurs, tous jeunes et sans doute dotés de bons appétits, n'avaient de vivres que pour quelques jours, et n'en n'ayant pas trouvé à acheter dans les îles, ne pouvaient se consoler que du souvenir des agapes anglaises tout en philosophant sur les «mutations de Fortune".

Nature n'est pas seulement celle qui oppose aux désirs humains la force des éléments contraires, elle présente également des dangers, tout aussi évidents, du côté des animaux. La surprise de Schaschek est manifeste en ce qui concerne l'absence des loups en Occident. Il est vrai que nous sommes avant la triste fin du Téméraire. Mais il insiste sur l'abondance du gibier dans les parcs royaux anglais; sans doute Pawlowsky, après lui, en rajoute-t-il, par Bède interposé, il note ce qu'il accepte comme un caractère intrinsèque de l'ile : "cette terre ne nourrit pas les loups. Et si l'on en introduit un, il meurt immédiatement. ce qui est prouvé par l'usage et l'expérimentation ${ }^{26} \%$. Pas de loups non plus en Bretagne, mais en raison de l'intervention humaine: «si l'on en prend un, sur le bord de la route, comme les larrons, il est pendu, sans même être écorchée ${ }^{2 i}$ ». Quel gaspillage, pour nos gens de l'Europe de la fourrure....

Si les régions froides, en fait, ne connaissent pas vraiment d'animaux dangereux, il n'en est pas de même des régions chaudes. Espagne et Portugal sont envahis par une étrange faune. Dans les montagnes qui entourent Ségovie, «il y a une grande quantité de lézards et de scorpions». Surtout, entre Castille et Portugal, dans les montagnes "hautes et rocheuses", se trouvent de "grandes quantités de serpents, de scorpions et de lézards". Les serpents sont d'étranges hybrides, ailés comme les chauve-souris et cornus, les scorpions, tout tachetés, ont la taille d'un chien de chasse cependant moyen, les lézards, verts, sont gros comme des chats. Mais plus que par leur horrible grosseur, ces animaux sont dangereux par leurs piqûres et morsures : ils s'attachent à vous avec opiniâtreté, lancent loin leur venin et vous tuent sur le champ. Deux moyens de limiter les risques pris : la possession d'antipoison à verser immédiatement sur les plaies (un «thériaque») et, surtout, passer quand ces bêtes, accablées par la chaleur, se réfugient sous les pierres et dans les grottes; autrement dit voyager au plus fort de la fournaise, soit, pour nos malheureux pèlerins, à midi, au mois de juillet...

Nous avons fait en sorte, en vérité, de traverser ces montagnes au plus vite, tâchant de nous réfugier dans les auberges quand la chaleur diminuait, par crainte de ces insectes ${ }^{28}$.

Pis, ces animaux ne s'attaquent qu'aux hommes et aux animaux domestiques, et laissent en paix les bêtes sauvages. Pour nos voyageurs, en particulier Schaschek, s'ajoute à ces petits monstres mortels l'épreuve des odeurs. Son récit est tout à fait sensible aux plantes, aux cultures et aux paysages. Pour notre propos, je crois qu'il faut en retenir l'ivresse des émanations poivrées, fort peu favorable aux longues chevauchées.

${ }^{26}$ Idem, p. 41.

${ }^{27}$ Idem, p. 44.

28 Idem, p. 67. 
Nous souffrions presque tous de maux de tête. dus à la très puissante odeur de ces arbres [les buis], odeur qu'ils répandaient alors très désagréablement à nos narines, par ces temps d'alternance de pluie et de sécheresse ${ }^{29}$,

se rappelle-t-il de sa traversée de la Biscaye. Et plus loin :

dans ces montagnes poussent des arbres. ...qui produisent des feuilles semblables à l'herbe de pyrèthre, et ils portent des fruits qui sentent très fort quand on les froisse $e^{30}$.

\section{Il est vrai que d'autres parfums sont mieux appréciés, tel celui des}

arbustes pas très hauts qui produisent des fleurs semblables aux roses, répandant un parfum qui vient des feuilles et non des fleurs ${ }^{3 !}$.

sans doute des cistes. Donc, une nature qui au fur et à mesure que l'on s'éloigne des pays connus devient de plus en plus incertaine, inattendue, pousse à sortir de ses habitudes, à mieux regarder, à s'adapter. Mais aussi une terre belle, qu'elle soit travaillée par l'homme, et nous avons une évocation des clôtures bretonnes $^{32}$, ou œuvre du créateur dans un Portugal idyllique ${ }^{33}$.

\section{Les hommes aussi peuvent être dangereux}

Les récits de nos voyageurs véhiculent un certain nombre d'idées reçues, sorties d'une longue tradition de catégorisation des peuples, dont l'origine se perd dans le fond des histoires, et peut-être des pré-histoires. Quelques pages après avoir vanté l'accueil des Anglais: «en aucun autre pays, nous n'avons reçu autant d'honneurs que là ${ }^{34}$ », Schaschek ajoute :

plus de chose à dire au sujet des Anglais que ce que j'en dit, je n'en n'ai pas, sinon que ce sont des gens, selon ce que j'ai vu, infidèles et rusés, ourdissant la mort des étrangers ; on peut croire qu'ils s'inclinent en pliant le genou, mais on ne doit pas leur faire confiance ${ }^{35}$,

ce qui semble un pure réminiscence de lecture, en contradiction avec tout son récit.

${ }^{29}$ Idem, p. 56.

${ }^{30}$ Idem, p. 68. Le pyrèthre est un insecticide à l'odeur forte, soufrée, obtenu en pulvérisant les capitules de certaines composées.

31 Idem, p. 59.

32 Idem, p. 44 : "le paysan a souvent entouré son champ de pierres, ce qui ne rend pas nécessaire de rester dans les pacages avec les troupeaux qui y séjournent, ni de les garder; nul dommage ne peut être causé aux voisins".

${ }^{33}$ Idem, p. 68 : « au pied même des montagnes, il y a un grand nombre de plantes qui portent une sorte de fraises, appelées fraises de mer. L'été où nous y étions, il y en avait des quantités et chacun pouvait les cueillir sans aucune interdiction. Ce pays abonde en figues, amandes et vin de raisins secs, que nous appellons chez nous, vin grec ».

${ }^{34}$ Idem, p. 37 : «c'est l'usage dans cette ville (Londres), si quelque hôte étranger de marque arrive, que les jeunes filles et les femmes viennent à lui à l'auberge. l'accueillent et lui apportent des cadeaux. Tout cela fut fait aussi pour nous ".

${ }^{35}$ Idem, p. 41. 
De même, les Basques et Béarnais semblent décrits, quoiqu'avec moins de crudité, d'après le Guide du Pelerin : sauvages et belliqueux ${ }^{36}$; et comme Schaschek est sensible au beau sexe, il note d'abord l'étrangeté de leurs femmes :

nous avons vu des femmes et des jeunes filles qui allaient la tête rasée... [elles] portent de si étranges costumes que je n'ai rien vu de semblable dans aucune des régions où nous avons voyage ${ }^{37}$.

Les dangers que l'on rencontre en fréquentant les hommes viennent cependant moins de leur propre nature que de l'ignorance des voyageurs, qui accumulent les maladresses et se jettent eux-mêmes au devant des pires difficultés. C'est la sagesse qu'acquiert notre garçon, au prix de salutaires frousses.

Avec l'arrogance de la jeunesse et de la fortune, nos chevaliers ont commencé en fanfare: ayant très glorieusement traversé le margraviat de Brandebourg en faisant étalage de leurs talents de tournoyeurs, ils arrivent en Palatinat. N'oublions pas leur équipage: une quinzaine de seigneurs et pages ${ }^{38}$, une dizaine de serviteurs, une soixantaine de chevaux, un chariot. En somme, une compagnie, une route. Pour ceux qui les croisent, sont-ils brigands ou pèlerins, chevaliers errants, écorcheurs ou ambassadeurs? Tout farauds de leur brillant passage à Anspach, les voici qui arrivent à Heidelberg; ils suspendent à leur cou leur signum, sans doute leurs armoiries, af in de proposer une joute à la cour du comte palatin du Rhin, Frédéric. Celui-ci ne les reçoit pas en personne, et il n'y aura pas de joutes. Schaschek avoue: «je n'ai pas compris les raisons de ce refus ${ }^{39}$ ". Tetzel, plus fin et expérimenté, lui, a saisi :

Monseigneur fut informé que le Palatin avait reçu cela [l'affichage des signa] comme une grave offense et avait cru que Monseigneur avait fait cela en pensant que le Palatin n'avait personne capable de lutter et de jouter contre les gens de Bohême.

Ils ne furent donc pas admis à la cour, bien que le comte se soit chargé de toutes leurs dépenses, ce qui était encore plus vexant pour Léon et sa compagnie, assimilés à des vagabonds auxquels on ferait l'aumône.

Difficultés à nouveau en Gueldre. Là. il fallut apprendre à louvoyer entre les partisans du duc de Bourgogne et ceux d'Adolphe de Gueldre qui venait de jeter son propre père en prison (1465). "Nous avons eu une traversée [du pays] difficile. De fait, de part et d'autre, il nous fut nécessaire d'obtenir publiquement une loyale protection ${ }^{40} "$.

A partir du moment où les interprètes deviennent obligatoires, les déplacements sont encore plus hasardeux. Je n'insisterai pas sur cet aspect

${ }^{36}$ Le guide du pélerin de Saint-Jacques de Compostelle. éd. J. Vielliard, Paris, 1944, p. 29 : «peuple barbare, différent de tous les peuples et par sa race plein de méchanceté ».

${ }^{37}$ Commentarius, p. 54-55.

38 Voir la liste donnée par F. Colette : «Le voyage en Europe du seigneur Léon de Rosmital en l'an 1466 ", dans Les chemins de Saint-Jacques, et la culture européenne, Campus stellae I, 1991, pp. 91-92.

39 Commentarius, p. 15.

${ }^{40}$ Idem, p. 20. 
linguistique ${ }^{41}$, mais il est certain que la sécurité des voyageurs était soumise aux capacités de persuasion et à la fidélité des " hérauts" et autres truchements. Ainsi quand, entre deux tempêtes, les seigneurs de Bohême furent pris sous le feu d'un navire anglais espérant couler les Français: "Monseigneur, comprenant qu'ils voulaient notre mort... ordonna à un héraut de monter à leur bord et de parler avec eux à la façon des hérauts ». La discussion se poursuivit sur le navire des pèlerins, après que les marins eurent amené les voiles ${ }^{42}$. Le sauf-conduit du roi d'Angleterre fit merveille, selon Schaschek; et encore plus pour Tetzel qui évoque une petite bataille navale avec tirs réciproques et note :

ils nous abordèrent et nous firent prisonniers une demi-journée, en installant de leurs hommes à notre bord... Nos marins furent courageux en exposant leurs vies et leurs personnes, mais si Monseigneur n'avait pas été présent, ils auraient perdu leurs vies et leurs biens ${ }^{43}$.

Les risques que firent courir les corsaires ne furent rien à côté des menaces des sauvages ibériques. Schaschek les juge systématiquement inamicaux, susceptibles et belliqueux, et surtout il les qualifie fréquemment d'infidèles, de païens. Seules la réflexion et la sagesse, ici souvent évidentes, du Seigneur Léon évitèrent les drames.

Peu accueillants, les gens de Biscaye refusèrent de considérer nos seigneurs comme des pèlerins exempts de droits: ils empruntaient un chemin non conventionnel. Aussi, entre Bilbao et Valmazeda. ce fut lincident. A un passage de pont, protégé par une tour, les péagers exigèrent leur dû.

Nous refusâmes de payer le passage. parce qu'en aucun autre lieu on ne l'avait exigé auparavant. Les percepteurs saisirent nos chevaux qui, en tête, portaient nos bagages, et accourant de la tour, ils voulurent nous tuer.

La bagarre éclata : "nous de sortir nos arbalètes et de les tourner vers eux ". Raisonnablement, Léon préféra payer le péage et recevoir des officiers basques des lettres scellées. Officiers ou bandits?

Il y en eut un qui nous raconta ensuite qu'ils avaient décidé, si l'un d'eux recevait une blessure, de nous exterminer tous. de saisir ce que nous avions dans nos bourses et nos sacs, pour l'acquittement du péage ${ }^{+1}$.

A quoi eut servi aux morts de verser ce droit, ne semble pas préoccuper le petit Schaschek.

Méconnaissance des usages et incompréhension furent aussi à l'origine des très mauvais rapports des voyageurs et de la population d'Olmedo. Nos garçons furent très critiques devant les moeurs « islamisés" des Castillans. Déjà, dans un

${ }^{41} \mathrm{Ph}$. Contamine, "L'hospitalité dans l'Europe du milieu du $X V^{\mathrm{c}}$ siècle, aspects juridiques, matériels et sociaux, d'après quelques récits de voyage ", La conscience européenne aux $X V$ et $X V T^{e}$ siècles, Actes du colloque international organisé à l'E.N.S.J.F. (30 sept. - 3 oct. 1980), Paris, 1982, pp. 75-87.

${ }^{42}$ Commentarius, p. 42.

${ }^{43}$ Des bömischen Hern Leo's... Reise, p. 47.

${ }^{44}$ Commentarius, p. 55. 
souci de légitimité, Léon avait fait choix de venir voir en premier le roi Henri IV, contre lequel, depuis l'année précédente, s'était révolté son frère Alonso; option qui provoqua l'animosité des partisans du prince rebelle. Les habitudes différentes, soulignées avec horreur par Schaschek ${ }^{45}$ finissent par causer de véritables émeutes. "Quand l'un de nous sortait de la maison, ils lui jetaient des choses, crachaient sur lui et se conduisaient de manière injurieuse ${ }^{46} "$. Pourquoi ces manifestations ? Nos héros présentaient une allure pour le moins étrange aux yeux des indigènes (incolae) : ils étaient de taille gigantesque et vêtus à la mode d'Europe centrale, avec des vêtements assez lourds et, surtout, ils portaient fièrement de longues boucles, jusqu'à la taille. L'étrangeté du pays et des visiteurs était vécue de chaque côté avec hostilité. C'était une attitude bien éloignée de celle des Londoniens, ou tout au moins ressentie par les voyageurs de façon toute différente. A Londres en effet,

on disait que l'on n'avait jamais vu personne portant des cheveux aussi longs et aussi beaux que les nôtres, et, naturellement. on disait qu'ils étaient attachés avec de la colle. Si l'un de nous se montrait ainsi, tête nue, il avait plus de spectateurs que s'il exhibait quelqu'animal exotique ${ }^{47}$.

A Olmedo: «ils cherchaient des prétextes pour nous voler plus commodément ce que nous avions, et pire à nous tuer ».

Ces intrus slaves, aux longs cheveux de femmes, n'en sont pas moins des hommes, ce qui aggrave leur cas. Ils se conduisent mal avec les servantes, tirent les oreilles et bottent les fesses du consommateur autochtone, indigné par leurs privautés et qui les a maudit en son langage. Une seule riposte pour le fier offensé, dans la grande tradition des épopées méditerranéennes: «deux heures après, ayant rassemblé environ quatre cent individus, il assaillit notre demeure, cherchant à nous $\operatorname{tuer}^{48}$ ". Seule la médiation des nobles envoyés par le roi permit d'apaiser les esprits.

Dans ce milieu hostile, le moindre geste pouvait entraîner un drame: en Galice, passant dans une châtaigneraie, le page de Léon se fit une fronde et mitrailla, semble-t-il au hasard, dans les frondaisons. Son excuse? Il l'avait vu faire aux indigènes. Bien évidemment, sous les arbres dormait un paysan qui fut blessé, le sang avait coulé... L'interprète semblait avoir apaisé les choses, et les pèlerins purent repartir et aller à Compostelle. Mais, en s'en retournant ils repassèrent par cet endroit, c'était défier les paysans. A l'auberge. ils furent cernés par « une centaine de gens armés d'épées, de lances, d'arcs, et arquebuses». Ils virent assurément leur dernière heure venue et, sanctifiés par l'accomplissement du pèlerinage, ils se serrèrent autour de Léon qui les harangua, en bon chef de guerre: "s'ils nous tuent, nos noms et la gloire de notre courage, resté ferme jusqu'au bout, seront éternels". Il fallait bien cependant revenir au pays pour pouvoir conter l'aventure, les assaillants,

\footnotetext{
45 « Pendant l'élévation, ils restent debout, comme des bêtes brutes », et encore : «ils mènent des vies si impures et sodomites que je n'oserai et j'aurai honte de raconter leur scélératesse.. » etc., Idem, p. 62. Le Guide du pèlerin est d'une crudité plus grande encore, op. cit., p. 29.

${ }^{46}$ Ibidem.

${ }^{47}$ Commentarius, p. 37.

${ }^{48}$ Idem, p. 36.
} 
sensibles au discours de l'interprète et mesurant les représailles qui les menaçaient s'ils tuaient la compagnie de «ce seigneur qui est de noble et haute maison et voyage pour rendre visite aux cours des rois les plus illustres ${ }^{49}$ ", laissèrent passer la troupe en route vers le roi de Portugal.

Au fur et à mesure que s'accumulaient les expériences, les discours se faisaient plus habiles, l'usage des interprètes était de plus en plus subtil, la diplomatie ne se développait pas en direction des seules puissants, rois, princes et évêques, mais se montrait plus conciliante envers les simples habitants.

Schaschek, sensible aux attaques physiques, en bon fils de chevalier, l'était moins aux conditions pratiques et économiques: quand il y a banquet on se gave, voire on se saoule; quand il n'y a rien, on s'en contente. En revanche, le bourgeois de Nuremberg se révèle plus soucieux du confort. de la nourriture de chaque jour et, de façon paradoxale, du décorum quotidien. En ce domaine également, les Espagnols lui semblent avoir une conduite inqualifiable : entre Burgos et Ségovie, pas d'auberge et partant pas de cuisiniers, à l'exception de celui de Léo, puisque nos gens ont depuis Calais renvoyé leurs serviteurs. Alors les voici vraiment chevaliers errants, fourrageant pour leurs montures, pétrissant leur pain, écorchant leur viande et ayant dû acheter jusqu'à leurs marmites. «Mon opinion est que partout au monde les tziganes sont mieux traités que nous ne le fûmes ". Vision assez drolatique de notre seigneur de Rozmital et Blatna vivant à la bohémienne sur les routes d'Espagne: "nous devions nous ménager un abri sous un arbre et attacher nos chevaux près de nous, comme des gitans... Monseigneur en prenait sa part, comme les autres ».

\section{Face à soi-même}

La dernière découverte est celle de la connaissance de soi, de ses propres limites, tout comme de celles d'autrui. Revenons sur les qualités propres de ces garçons : de leur mission politique, nous ne saurons rien directement, comme il est naturel pour des semi-diplomates ; en revanche, nous ne pouvons ignorer leur force physique et l'étalage qu'ils en font. Au début, partout où ils passent ils exhibent leurs talents équestres, leurs muscles et leur valeur. Ils pratiquent le tournoi à cheval, divers jeux montés, mais aussi des combats de puissance et de tactique : une sorte de lutte $^{50}$. Pour attirer les compétiteurs, ils commençaient par faire des démonstrations entre eux, puis cherchaient des champions. A Anspach, à Cologne, à Bruxelles, ils ont fait merveille. Le plus habile est certainement le comte Jean de Zahrosky, un géant qui provoquait l'admiration; aussi bon à cheval que dans la lutte, il restait en selle en fonçant contre un mur, lance pointée, sous les yeux sidérés de toute la cour

\footnotetext{
49 Ibidem.

${ }^{50}$ En Bohème, cette lutte devait se pratiquer presque nu. ce qui était inconvenant pour les dames spectatrices. C'est tout au moins ce qui apparait dans les règlements de Bourgogne où l'on doit être revêtu d'un pourpoint et de chausse afin " que nul ne rende inconvenante l'entrée des dames et des demoiselles I».
} 
de Bourgogne qui pourtant ne manquait pas de spectacles. Schaschek est intarissable :

il frappa le mur si fort que le cheval en fut assis sur sa croupe. Là-dessus, les gens de la cour du duc vinrent voir si le seigneur Jean n'aurait pas été lié sur son cheval, puisqu'un coup si violent ne l'avait pas désarçonné.

Ayant recommencé l'épreuve avec succès, Jean passa pour «appartenir à la race antique des Géants », on venait soupeser ses armes à l'auberge, et le vieux duc Philippe le Bon, lui-même aux portes d'une mort dont il avait si peur, leur déclara : «vous jouez vos vies, comme si vous ne vouliez point vivre ${ }^{51}$ ". Ce qui est bien le propre de la jeunesse.

Pour cela aussi, l'Espagne du pèlerinage fut la pierre de touche de l'or ou du doré. Le valeureux Jean de Zahrovsky continuait à faire le faraud : c'est lui qui en jouant «effleura le sein d'une servante", provoquant l'émeute que l'on sait. Il était pourtant près de recevoir une leçon. Au cours de ce qui devait être pour lui une démonstration de pure forme, à la cour du roi de Castille, il fut d'abord effectivement vainqueur d'un petit homme râblé. Mais la seconde manche le retrouva à terre. Jean, projeté par dessus l'épaule du petit Hercule sans avoir le temps de se reconnaître, avait trouvé son maître, à la satisfaction bruyante et insolente des spectateurs autochtones $^{52}$.

Le retournement fut plus complet encore, et saint Jacques y fut pour quelque chose. Aux portes de Compostelle, la ville de Padron passait pour le lieu où l'apôtre aurait commencé l'évangélisation de l'Espagne, avant son retour à Jérusalem, son martyre et sa navigation miraculeuse vers le champ de l'Etoile. Persécuté par les païens de Padron, Jacques se réfugiait, disait-on, dans une grotte, devenue une des excursions possibles pour ceux qui abordaient Compostelle par le sud. L'accès en était une étroite fente; à l'intérieur, où sourdait une fontaine, il régnait la fraîcheur. Schaschek raconte:

celui qui entre dans la grotte avec humilité est absout de bien des péchés. Moi, j'y suis entré avec Burian. Knesekius et son frère. Petipetski, et Miros. Jean de Zehrovsky s'y évanouit et gisait, terreux et sans force, et nous le sortimes à grandpeine tant l'entrée était étroite ${ }^{53}$.

Vaincu par la différence de température, et la fatigue, le géant s'abattit, il était allé au bout du chemin de l'humiliation, chemin salutaire. Sur la route du retour, il fut à nouveau vainqueur, mais un vainqueur sans gloriole. La leçon n'était pas seulement valable pour lui, tous terminèrent le périple en ayant appris la vanité de la gloire humaine.

${ }^{51}$ Commentarius, p. 25.

52 Qui magna laeticia exultantes, victoriam ei (leur champion) gratulabantur, Idem, p. 62.

53 Idem, p. 72. Ses compagnons, prudents, interdirent à Léon l'entrée de la grotte! 
Le monde auquel se confrontent ces voyageurs est aussi dangereux par son essence que par l'ignorance de ceux qui le parcourent. Cet univers hostile peut néanmoins révéler ce qui se cache derrière l'apparence des êtres et des choses. Les seigneurs pèlerins devaient arriver à la tombe du saint en ayant été jusqu'au bout de leurs peurs, en ayant côtoyé la mort, réelle ou imaginaire, vécu la pauvreté, assumé l'humiliation. C'est à ce prix que leur «heureux voyage " devint un vrai pèlerinage.

Françoise Michaud-Fréjaville (Université d'Orléans) 